\title{
SENDOV CONJECTURE FOR HIGH DEGREE POLYNOMIALS
}

\author{
JÉRÔME DÉGOT \\ (Communicated by Richard Rochberg)
}

\begin{abstract}
Sendov's conjecture says that if all zeros of a complex polynomial $P$ lie in the closed unit disk and $a$ denotes one of them, then the closed disk of center $a$ and radius 1 contains a critical point of $P$ (i.e. a zero of its derivative $P^{\prime}$ ). The main result of this paper is to prove that, for each $a$, there exists an integer $N$ such that the disk $|\zeta-a| \leq 1$ contains a critical point of $P$ when the degree of $P$ is larger than $N$. We obtain this by studying the geometry of the zeros and critical points of a polynomial which would eventually contradict Sendov's conjecture.
\end{abstract}

\section{INTRODUCTION}

Sendov's conjecture can be stated as follows:

Conjecture (Sendov). Let $P(z)=(z-a) \prod_{k=1}^{n-1}\left(z-z_{k}\right)$ be a monic complex polynomial having all its zeros in the closed unit disk. Then, there exists a zero $\zeta$ of the derivative $P^{\prime}$ such that $|\zeta-a| \leq 1$.

This conjecture appears for the first time in 1967 Hayman's book Research Problems in Function Theory [3], where it was improperly attributed to the Bulgarian mathematician Illief. Since 1967, it has been proved for a few special cases, for example: polynomials having at most 8 distinct zeros [1], when $|a|=1[$ ], if $P$ vanishes at 0 , when all the summits of the convex hull of the zeros of $P$ lie on the unit circle [7, but the general case is still open in spite of 80 papers devoted to it. Surveys of the problem have been given by M. Marden [4] and Bl. Sendov [8], and we refer the reader to these for further information and bibliographies.

Set $P(z)=(z-a) \prod_{k=1}^{n-1}\left(z-z_{k}\right)$ with $a$ real, $0<a<1$ and $\left|z_{k}\right| \leq 1$ for all $k=1, \ldots, n-1$. We prove in this paper that the derivative $P^{\prime}$ has a zero in the disk $|\zeta-a| \leq 1$ when $n$ is larger than an integer bound $N$. Where the bound $N$ depends on $a$, unfortunately we will not be able to give an explicit formula for $N$ but just describe how it can be computed.

Assuming that $P$ contradicts Sendov's conjecture, we will estimate below and above the positive real number $|P(c)|$ for some real $c$ satisfying $0<c<a$. This leads to a contradiction when the degree $n$ of the polynomial $P$ is large enough.

\section{EXCLUSION DOMAIN}

Let us introduce two useful theorems in geometry of polynomials. The first one is due to Q. I. Rahman and G. Schmeisser (see [5, p. 100]).

Received by the editors November 16, 2011 and, in revised form, May 18, 2012.

2010 Mathematics Subject Classification. Primary 30C10, 30C15; Secondary 12D10.

Key words and phrases. Sendov conjecture, polynomial, geometry of polynomial, zeroes, inequalities.

(C)2014 American Mathematical Society 1337

Reverts to public domain 28 years from publication 
Theorem 1. Let $P$ be a complex polynomial of degree $\leq n$, and $\delta$ a complex number satisfying $P^{\prime}(\delta) \neq 0$. For any $\omega \in \mathbb{C}$ different from $P(\delta)$, the polynomial $P(z)-\omega$ has a zero in the disk whose diameter is the line segment $\left[\delta, \delta-\frac{n(P(\delta)-\omega)}{P^{\prime}(\delta)}\right]$.

The second one is due to J. H. Grace (see [2, p. 356]); we will call it the Perpendicular bisector theorem.

Theorem 2. Let $P$ denote a polynomial, and $\alpha$ and $\beta$ two complex numbers such that $P(\alpha)=P(\beta)$. Then, the perpendicular bisector of the line segment $[\alpha, \beta]$ intersects the convex hull of the zeros of $P^{\prime}$ (i.e. each half-plane delimited by the bisector contains at least one zero of $\left.P^{\prime}\right)$.

The following theorem gives an exclusion domain (i.e. without zero) for a polynomial $P$ that contradicts Sendov's conjecture.

Theorem 3. Let $P(z)=(z-a) \prod_{k=1}^{n-1}\left(z-z_{k}\right)$, where $a$ is a real, $0<a<1$ and $\left|z_{k}\right| \leq 1$ for $k=1, \ldots, n-1$. Suppose that $P^{\prime}(\zeta) \neq 0$ for $|\zeta-a| \leq 1$. Then, for any $c \in(0, a)$, the polynomial $P$ cannot have any zero in the disk of center $c$ and radius $1-\sqrt{1-c(a-c)}$.

Proof. On the contrary, assume that there exists a zero $\gamma$ of $P$ such that $|c-\gamma| \leq$ $1-\sqrt{1-c(a-c)}$. Suppose that $\Im(\gamma) \leq 0$ and let $\xi$ denote the complex number, with positive imaginary part, satisfying

$$
|\xi-a|=|\xi|=1,
$$

so $\xi$ is at the intersection of circles of radius 1 , centered at $a$ and 0 . We have

$$
|\xi-\gamma| \leq|\xi-c|+|c-\gamma| \leq 1=|\xi-a| \cdot
$$

Therefore, the perpendicular bisector of the line segment $[\gamma, a]$ doesn't intersect the "lunula region" $\mathcal{L}$ defined by

$$
\mathcal{L}=\{z \in \mathbb{C} ;|z| \leq 1 \text { and }|z-a| \geq 1\}
$$

By the Gauss-Lucas theorem and hypothesis, $\mathcal{L}$ contains all the critical points of $P$. Since $P(\gamma)=P(a)$, Theorem 2 implies that the perpendicular bisector of the line segment $[\gamma, a]$ should intersect the convex hull of the critical points and then the region $\mathcal{L}$. This leads to a contradiction and proves the theorem.

\section{LEMMAS}

We give in this section some technical inequalities. For the convenience of the reader the proofs are deferred to the end of the paper (section 7 ).

Lemma 1. Let $\delta \in(0,1)$ and $z_{1}, \ldots, z_{n}$ be complex numbers which all lie in the closed unit disk. Writing $m:=\frac{1}{n} \Re\left(\sum_{k=1}^{n} z_{k}\right)$, we have

$$
\prod_{k=1}^{n}\left|\delta-z_{k}\right| \leq\left(\sqrt{1+\delta^{2}-2 \delta m}\right)^{n} \text {. }
$$

Lemma 2. Let $\delta$ and $a$ be real numbers such that $0<\delta<a<1$ and let $\zeta_{1}, \ldots, \zeta_{n-1}$ denote complex numbers satisfying $\left|\zeta_{k}\right| \leq 1$ and $\left|\zeta_{k}-a\right|>1$, for $k=1, \ldots, n-1$. Writing $s:=\frac{1}{n-1} \Re\left(\sum_{k=1}^{n-1} \zeta_{k}\right)$ and $q:=\frac{a / 2-s}{1+a / 2}$, we have

$$
\prod_{k=1}^{n-1}\left|\frac{\delta-\zeta_{k}}{a-\zeta_{k}}\right| \leq\left\{\left(\frac{1+\delta}{1+a}\right)^{q}{\sqrt{1+\delta^{2}-\delta a}}^{1-q}\right\}^{n-1} .
$$


Lemma 3. Let $\delta$, $a$ and $b$ be real numbers such that $0<\delta<a<1<b$ and let $\xi_{1}, \ldots, \xi_{n-1}$ denote complex numbers satisfying $\left|\xi_{k}\right| \leq 1$ and $\left|\xi_{k}-a\right|>1$, for $k=1, \ldots, n-1$. Set

$$
s:=\frac{1}{n-1} \Re\left(\sum_{k=1}^{n-1} \xi_{k}\right)<\frac{a}{2}
$$

and

$$
p:=\frac{a / 2-s}{1-a / 2}, \quad q:=\frac{a / 2-s}{1+a / 2}, \quad B_{1}:=(1+b-a)^{p}\left(\sqrt{1+b^{2}-b a}\right)^{1-p}
$$

and $B_{2}:=(1+b)^{q}\left(\sqrt{1+b^{2}-b a}\right)^{1-q}$. We have

$$
\prod_{k=1}^{n-1}\left|b-\xi_{k}\right| \geq \min \left(B_{1}, B_{2}\right)^{n-1}
$$

Remark. The important point is that $\min \left(B_{1}, B_{2}\right)>\sqrt{1+b^{2}-b a}$.

Lemma 4. Let $c$ and $r$ denote real numbers such that $0<c<1$ and $0<r<1-c$. Assume that $z_{1}, \ldots, z_{n}$ are complex numbers satisfying $0<\left|z_{k}\right| \leqslant 1$ and $\left|\frac{c-z_{k}}{1-c z_{k}}\right| \geq r$, for $k=1, \ldots, n$. We have

$$
\prod_{k=1}^{n}\left|\frac{c-z_{k}}{1-c z_{k}}\right| \geq r^{\beta}, \quad \text { where } \beta:=\frac{\sum_{k=1}^{n} \log \left|z_{k}\right|}{\log \left(\frac{c+r}{1+c r}\right)} .
$$

\section{UpPer estimation of $|P(c)|$}

Theorem 4. Let $P(z)=(z-a) \prod_{k=1}^{n-1}\left(z-z_{k}\right)$, where $0<a<1$ and $\left|z_{k}\right| \leq 1$ for $k=1, \ldots, n-1$. Assume that $P^{\prime}(\zeta) \neq 0$ for all $|\zeta-a| \leq 1$. Then, for any real $\delta \in(0, a)$, we have

$$
\left|\frac{P(\delta)}{P^{\prime}(a)}\right| \geq \frac{1-\sqrt{1+\delta^{2}-\delta a}}{n}
$$

Proof. Write $R=\frac{n P(\delta)}{P^{\prime}(a)}$ and denote by $\xi$ the complex number satisfying $|\xi-a|=$ $|\xi|=1$ with positive imaginary part. Theorem 1 asserts that the disk of center $a$ and radius $|R|$ contains a complex number $\gamma$ such that $P(\delta)=P(\gamma)$; there is no loss of generality assuming $\Im(\gamma) \geq 0$. By Theorem 2 the perpendicular bisector of the line segment $[\delta, \gamma]$ intersects the convex hull of the zeros of $P^{\prime}$; therefore $|\xi-\delta| \geq|\xi-\gamma|$ (see Figure 1 below). We deduce that

$$
|R| \geq 1-|\xi-\gamma|
$$

and then

$$
\left|\frac{P(\delta)}{P^{\prime}(a)}\right| \geq \frac{1-\sqrt{1+\delta^{2}-\delta a}}{n} .
$$

Corollary 1. Let $P$ be defined as above, and put $m:=\frac{1}{n} \Re\left(a+\sum_{k=1}^{n-1} z_{k}\right)$. Then

$$
m \leq \inf _{\delta \in(0, a)}\left(\frac{\delta}{2}-\frac{1}{\delta n} \log \left(1-\sqrt{1+\delta^{2}-\delta a}\right)\right) .
$$




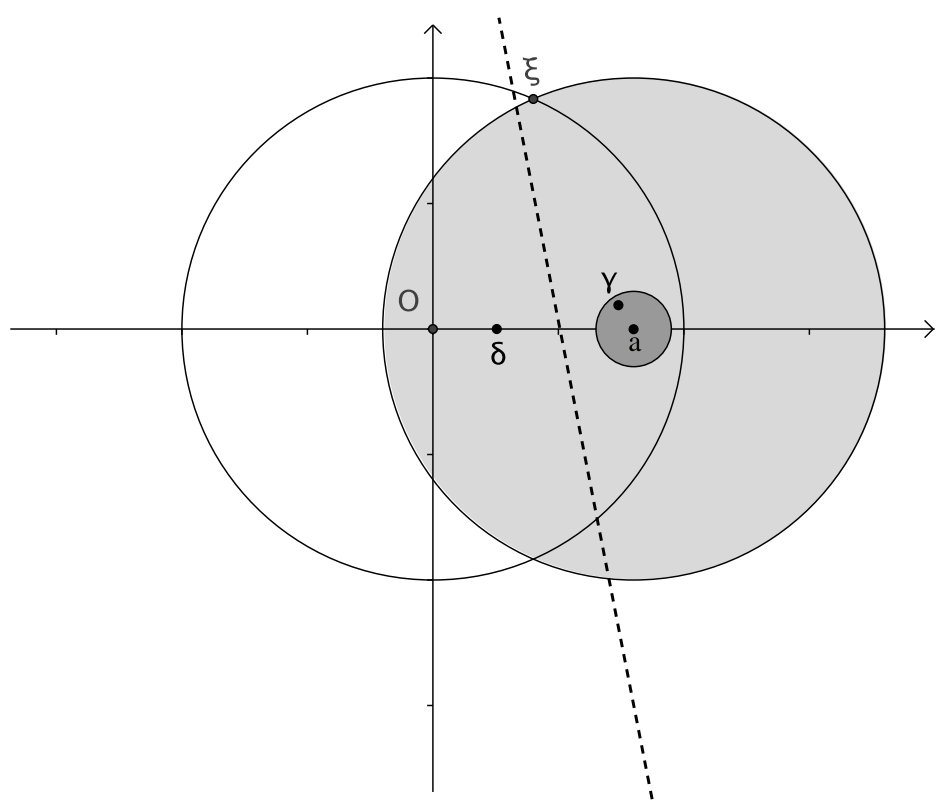

Figure 1. Illustration of the proof of Theorem 4

Proof. Let $\delta \in(0, a)$. Theorem 4 gives

$$
|P(\delta)| \geq \frac{1-\sqrt{1+\delta^{2}-\delta a}}{n}\left|P^{\prime}(a)\right|,
$$

and then $|P(\delta)| \geq 1-\sqrt{1+\delta^{2}-\delta a}$. By Lemma 1 we know that

$$
|P(\delta)| \leq\left(\sqrt{1+\delta^{2}-2 \delta m}\right)^{n} ;
$$

therefore

$$
1-\sqrt{1+\delta^{2}-\delta a} \leq\left(\sqrt{1+\delta^{2}-2 \delta m}\right)^{n}
$$

We deduce that

$$
\begin{aligned}
& \frac{1}{n} \log \left(1-\sqrt{1+\delta^{2}-\delta a}\right) \leq \frac{\delta^{2}-2 \delta m}{2} \\
\Longleftrightarrow & m \leq \frac{\delta}{2}-\frac{1}{\delta n} \log \left(1-\sqrt{1+\delta^{2}-\delta a}\right),
\end{aligned}
$$

which establishes the desired inequality.

Remark. When $n \rightarrow+\infty$, inf $_{\delta \in(0, a]}\left(\frac{\delta}{2}-\frac{1}{\delta n} \log \left(1-\sqrt{1+\delta^{2}-\delta a}\right)\right) \rightarrow 0$. Therefore, if $P$ satisfies the assumptions of Theorem 4 , then $m$ should be negative or close to zero for large $n$. This fact will be of crucial importance in section 5 to see that $K>1$.

Theorem 5. Let $P(z)=(z-a) \prod_{k=1}^{n-1}\left(z-z_{k}\right)$, where $0<a<1$ and $\left|z_{k}\right| \leq 1$ for all $k$. Suppose that the critical points $\zeta_{1}, \ldots, \zeta_{n-1}$ of $P$ all lie in $|z-a|>1$, which implies that $\Re\left(\zeta_{k}\right)<a / 2$ for $k=1, \ldots, n-1$, and so also

$$
s:=\frac{1}{n-1} \Re\left(\sum_{k=1}^{n-1} \zeta_{k}\right)<a / 2 .
$$


Let $q:=\frac{a / 2-s}{1+a / 2}$ and $N_{1}$ be the smallest integer such that

$$
\left(\frac{1+a / 2}{1+a}\right)^{q} \leq\left(\frac{1-\sqrt{1-a^{2} / 4}}{n a}\right)^{1 /(n-1)} \text { for all } n \geq N_{1}
$$

Then, if $n \geq N_{1}$, we have

$$
\left|P^{\prime}(a)\right| \leq \frac{16 n}{a^{2}} \quad \text { and } \quad|P(0)| \geq \frac{a^{2}}{16} .
$$

Proof. Assume that $n \geq N_{1}$. Lemma 2 asserts that for all $\delta \in(0, a / 2)$,

$$
\begin{aligned}
\left|\frac{P^{\prime}(\delta)}{P^{\prime}(a)}\right| & \leq\left[\left(\frac{1+\delta}{1+a}\right)^{q}{\sqrt{1+\delta^{2}-\delta a}}^{1-q}\right]^{n-1} \\
& \leq\left(\frac{1+a / 2}{1+a}\right)^{q(n-1)} \\
& \leq \frac{1-\sqrt{1-a^{2} / 4}}{n a} .
\end{aligned}
$$

We deduce that

$$
\begin{aligned}
\left|\frac{P(0)}{P^{\prime}(a)}-\frac{P(a / 2)}{P^{\prime}(a)}\right| & \leq \frac{a}{2} \sup _{\delta \in[0, a / 2]}\left|\frac{P^{\prime}(\delta)}{P^{\prime}(a)}\right| \\
& \leq \frac{1-\sqrt{1-a^{2} / 4}}{2 n}
\end{aligned}
$$

By Theorem 4, we know that

$$
\left|\frac{P(a / 2)}{P^{\prime}(a)}\right| \geq \frac{1-\sqrt{1-a^{2} / 4}}{n} ;
$$

then

We deduce the theorem.

$$
\left|\frac{P(0)}{P^{\prime}(a)}\right| \geq \frac{1-\sqrt{1-a^{2} / 4}}{2 n} \geq \frac{a^{2}}{16 n} .
$$

Remark. This theorem expresses that the zeros of $P$ should lie nearby the unit circle and those of $P^{\prime}$ close to the circle $|z-a|=1$ when the degree of $P$ is large enough.

We are now in a position to give an upper bound for $|P(c)|$.

Theorem 6. Let $P(z)=(z-a) \prod_{k=1}^{n-1}\left(z-z_{k}\right)$, where $0<a<1$ and $\left|z_{k}\right| \leq 1$ for all $k$. Suppose that the derivative $P^{\prime}$ doesn't vanish in the disk $|\zeta-a| \leq 1$. Let $q$ and $N_{1}$ be defined as in Theorem 5, $c \in(0, a)$ and set

$$
D:=\max \left\{\left(\frac{1}{1+a}\right)^{q} ;\left(\frac{1+c}{1+a}\right)^{q}\left(\sqrt{1+c^{2}-a c}\right)^{1-q}\right\}<1 .
$$

Define $\mathrm{N}_{2}$ to be the smallest integer such that

$$
D^{n-1} \leq \frac{a}{16 n} \quad \text { for all } \quad n \geq N_{2} .
$$

Then, if $n \geq \max \left\{N_{1}, N_{2}\right\}$ we have

$$
|P(c)| \leq 1+a .
$$


Proof. Consider the mapping $f$ defined by

$$
\begin{aligned}
f:[0, c] & \longrightarrow \mathbb{R} \\
x & \longmapsto \log \left[\left(\frac{1+x}{1+a}\right)^{q}{\sqrt{1+x^{2}-x a}}^{1-q}\right] ;
\end{aligned}
$$

the first derivative of $f$ is given by

$$
f^{\prime}(x)=\frac{x^{2}+(1-a+m) x-m}{(1+x)\left(1+x^{2}-x a\right)} .
$$

Since $f^{\prime}(x)$ shares the sign of $x^{2}+(1-a+m) x-m$, by Lemma 2 we have

$$
\left|\frac{P^{\prime}(\delta)}{P^{\prime}(a)}\right| \leq D^{n-1}, \quad \text { and then } \quad \sup _{\delta \in[0, c]}\left|\frac{P^{\prime}(\delta)}{P^{\prime}(a)}\right| \leq \frac{a}{16 n} .
$$

For all $n \geq \max \left\{N_{1}, N_{2}\right\}$, using Theorem 5, we deduce that

$$
|P(0)-P(c)| \leq \sup _{\delta \in[0, c]}\left|\frac{P^{\prime}(\delta)}{P^{\prime}(a)}\right| c\left|P^{\prime}(a)\right| \leq \frac{c}{a} \leq 1 .
$$

Therefore

$$
|P(c)| \leq 1+|P(0)| \leq 1+a
$$

\section{Lower estimation of $|P(c)|$}

We prove, in this section, that there exist constants $C>0$ and $K>1$ such that if $n$ is large enough the inequality $|P(c)| \geq C K^{n}$ holds. Three new lemmas are needed; their proofs are still deferred to the end (section 7).

Lemma 5. Let $h$ and $c$ be positive real numbers satisfying $0<c<1-h$. For all $z \in \mathbb{C}$, if $|z| \geq 1-h$, we have

$$
|c-z| \geq \frac{c}{1-h}\left|\frac{(1-h)^{2}}{c}-z\right| .
$$

Lemma 6. Let $P(z)=(z-a) \prod_{k=1}^{n-1}\left(z-z_{k}\right)$, where $0<a<1$ and $\left|z_{k}\right| \leq 1$ for $k=1, \ldots, n-1$. Denote by $\zeta_{1}, \ldots, \zeta_{n-1}$ the critical points of $P$. Then, for all $b>1$, we have

$$
(b-a) \prod_{k=1}^{n-1}\left|b-z_{k}\right| \geq(b-1) \prod_{k=1}^{n-1}\left|b-\zeta_{k}\right| .
$$

Lemma 7. Let $P(z)=(z-a) \prod_{k=1}^{n-1}\left(z-z_{k}\right)$, where $0<a<1$ and $\left|z_{k}\right| \leq 1$ for $k=1, \ldots, n-1$. Suppose that $P^{\prime}(\zeta) \neq 0$ for $|\zeta-a| \leq 1$. Let $c$ and $h$ be real numbers such that $0<h<c<a<1-h$. Then, the disk $\mathcal{D}$ defined by

$$
\mathcal{D}:=\left\{z \in \mathbb{C} ;\left|\frac{(c-z)}{(1-h)^{2}-c z}\right| \leq \frac{c(a-c)}{2\left((1-h)^{2}-c^{2}\right)}\right\}
$$

contains no zero of $P$.

We can now give a lower estimate of $|P(c)|$. 
Theorem 7. Let $P(z)=(z-a) \prod_{k=1}^{n-1}\left(z-z_{k}\right)$, where $0<a<1$ and $\left|z_{k}\right| \leq 1$ for $k=1, \ldots, n-1$. Suppose that $P^{\prime}(\zeta) \neq 0$ for $|\zeta-a| \leq 1$. Let $c \in(0, a), q$ and $N_{1}$ be defined as in Theorem 5, Set

$$
p:=\frac{a / 2-m}{1-a / 2}, \quad r:=\frac{c(a-c)}{2\left(1-c^{2}\right)}, \quad \alpha:=\log \left(\frac{a}{16}\right) / \log \left(\frac{c+r}{1+c r}\right)
$$

and

$$
K:=\min \left\{(1+c-a c)^{p}{\sqrt{1+c^{2}-a c}}^{1-p} ;(1+c)^{q}{\sqrt{1+c^{2}-a c}}^{-q}\right\} .
$$

If $n \geq N_{1}$, we have

$$
|P(c)| \geq \frac{(1-c)(a-c)}{1-a c} r^{\alpha} K^{n-1} .
$$

Remark. Observe that if $c$ is sufficiently close to $a$, then $K>1$.

Proof. Fix $0<h<1-a$ and assume that the zeros of $P$ are indexed such that for all $k \geq n_{0}$ we have $\left|z_{k}\right| \geq 1-h$. Let $b_{h}=\frac{(1-h)^{2}}{c}$; we have

$$
\begin{aligned}
|P(c)| & =|c-a| \prod_{k=1}^{n_{0}-1}\left|c-z_{k}\right| \prod_{k=n_{0}}^{n-1}\left|c-z_{k}\right| \\
& \geq(a-c) \prod_{k=1}^{n_{0}-1}\left|c-z_{k}\right|\left(\frac{c}{1-h}\right)^{n-n_{0}} \prod_{k=n_{0}}^{n-1}\left|b_{h}-z_{k}\right| \quad \text { (using Lemma 5) } \\
& =\left(\frac{a-c}{b_{h}-a}\right) \prod_{k=1}^{n_{0}-1}\left|\frac{c-z_{k}}{b_{h}-z_{k}}\right|\left(\frac{c}{1-h}\right)^{n-n_{0}}\left|b_{h}-a\right| \prod_{k=1}^{n-1}\left|b_{h}-z_{k}\right| .
\end{aligned}
$$

By Lemma 6, we deduce

$$
\begin{aligned}
|P(c)| & \geq\left(\frac{a-c}{b_{h}-a}\right) \prod_{k=1}^{n_{0}-1}\left|\frac{c-z_{k}}{b_{h}-z_{k}}\right|\left(\frac{c}{1-h}\right)^{n-n_{0}}\left(b_{h}-1\right) \prod_{k=1}^{n-1}\left|b_{h}-\zeta_{k}\right| \\
& \geq\left(\frac{\left(b_{h}-1\right)(a-c)}{\left(b_{h}-a\right)}\right) \prod_{k=1}^{n_{0}-1}\left|\frac{\left(c-z_{k}\right)(1-h)}{\left(b_{h}-z_{k}\right) c}\right|\left(\frac{c}{1-h}\right)^{n-1} \prod_{k=1}^{n-1}\left|b_{h}-\zeta_{k}\right| .
\end{aligned}
$$

Using Lemma 3, we obtain

$$
|P(c)| \geq\left(\frac{\left(b_{h}-1\right)(a-c)}{\left(b_{h}-a\right)}\right) \prod_{k=1}^{n_{0}-1}\left|\frac{\left(c-z_{k}\right)(1-h)}{\left(b_{h}-z_{k}\right) c}\right|\left(K_{h}\right)^{n-1},
$$

where $K_{h}$ is defined by

$$
\frac{c}{1-h} \min \left\{\left(1+b_{h}-a\right)^{p}{\sqrt{1+b_{h}^{2}-a b_{h}}}^{-p} ;\left(1+b_{h}\right)^{q}{\sqrt{1+b_{h}^{2}-a b_{h}}}^{-q}\right\} .
$$

Set $c^{\prime}=\frac{c}{1-h}, z_{k}^{\prime}=\frac{z_{k}}{1-h}$ and $r_{h}=\frac{c(a-c)(1-h)}{2\left((1-h)^{2}-c^{2}\right)}$; by Lemma 7 we know that

$$
\left|\frac{\left(c-z_{k}\right)(1-h)}{\left(b_{h}-z_{k}\right) c}\right|=\left|\frac{c^{\prime}-z_{k}^{\prime}}{1-c^{\prime} z_{k}^{\prime}}\right| \geq r_{h} .
$$

Lemma 4 gives that

$$
\prod_{k=1}^{n_{0}-1}\left|\frac{c^{\prime}-z_{k}^{\prime}}{1-c^{\prime} z_{k}^{\prime}}\right| \geq r_{h}^{\beta_{h}}
$$


where $\beta_{h}=\log \left(\prod_{k=1}^{n_{0}-1}\left|z_{k}^{\prime}\right|\right) / \log \left(\frac{c^{\prime}+r_{h}}{1+c^{\prime} r_{h}}\right)$. By Theorem 5, we have

$$
\prod_{k=1}^{n_{0}-1}\left|z_{k}^{\prime}\right| \geq \prod_{k=1}^{n_{0}-1}\left|z_{k}\right| \geq \prod_{k=1}^{n-1}\left|z_{k}\right| \geq \frac{a}{16}
$$

thus in (3) the exponent $\beta_{h}$ can be replaced by $\alpha_{h}=\log \left(\frac{a}{16}\right) / \log \left(\frac{c^{\prime}+r_{h}}{1+c^{\prime} r_{h}}\right)$. Combining (2) with (3), we deduce that

$$
|P(c)| \geq\left(\frac{\left(b_{h}-1\right)(a-c)}{\left(b_{h}-a\right)}\right) r_{h}^{\alpha_{h}}\left(K_{h}\right)^{n-1} .
$$

The theorem follows by letting $h \rightarrow 0$.

\section{Main Result And COMputations}

We can now formulate our main result.

Theorem 8. Let $P(z)=(z-a) \prod_{k=1}^{n-1}\left(z-z_{k}\right)$, where $0<a<1$ and $\left|z_{k}\right| \leq 1$ for $k=1, \ldots, n-1$. Let $N_{1}$ and $N_{2}$ be defined by Theorems 5 and 6 , and suppose that $c \in(0, a)$ is chosen sufficiently close to a to have $K>1$ ( $K$ is defined in Theorem (7). If

$$
\operatorname{deg}(P) \geq N:=\max \left\{N_{1} ; N_{2} ; \frac{\log \left(\frac{(1+a)(1-a c)}{(1-c)(a-c)}\right)-\alpha \log (r)}{\log (K)}+1\right\},
$$

then the derivative $P^{\prime}$ has a zero in the disk $|\zeta-a| \leq 1$.

Proof. Assume, on the contrary, that $P^{\prime}(\zeta) \neq 0$ for $|\zeta-a| \leq 1$. Combining the results of Theorems [6] and [7, we obtain

$$
1+a \geq|P(c)| \geq \frac{(1-c)(a-c)}{1-a c} r^{\alpha} K^{n-1} ;
$$

thus

$$
\log \left(\frac{(1+a)(1-a c)}{(1-c)(a-c)}\right) \geq \alpha \log (r)+(n-1) \log K,
$$

i.e.

$$
n \leq\left[\log \left(\frac{(1+a)(1-a c)}{(1-c)(a-c)}\right)-\alpha \log (r)\right] / \log (K)+1 .
$$

This implies the theorem.

Before turning to computations, let us recall the main formulae:

$$
\begin{aligned}
p & =\frac{a / 2-m}{1-a / 2} ; \\
q & =\frac{a / 2-m}{1+a / 2} ; \\
K & =\min \left\{(1+c-a c)^{p}{\sqrt{1+c^{2}-a c}}^{1-p} ;(1+c)^{q}{\sqrt{1+c^{2}-a c}}^{1-q}\right\} ; \\
r & =\frac{c(a-c)}{2\left(1-c^{2}\right)} \\
\alpha & =\log \left(\frac{a}{16}\right) / \log \left(\frac{c+r}{1+c r}\right) ; \\
N & =\max \left\{N_{1} ; N_{2} ; \frac{\log \left(\frac{(1+a)(1-a c)}{(1-c)(a-c)}\right)-\alpha \log (r)}{\log (K)}+1\right\} .
\end{aligned}
$$

Therefore, if $a$ and $c$ are given with $0<c<a$, to compute $N$ one needs to know $m$. Its exact value is unknown but can be replaced by the upper estimate given by 
Corollary 1, which depends only on $n$. Computations can be done in the following way; assume that $a \in(0,1)$ is given:

(i) Choose arbitrarily $c \in(0, a)$ and $m>0$.

(ii) Compute $p, q, K$ and check that $K>1$. If not, go back to the first step, increasing $c$.

(iii) Compute $N$ and deduce the upper estimate of $m$ given in Corollary 1 , If it is larger than $m$, go back to the first step, increasing $m$, otherwise decreasing $m$, and repeat until equality holds.

(iv) Adjust the choice of $c$ to obtain the smallest value of $N$.

Finally, find below the values of $N$ obtained for many choices of $a$.

\begin{tabular}{|c|c|c|c|c|c|c|c|c|}
\hline$a$ & $c$ & $m$ & $r$ & $\alpha$ & $p$ & $q$ & $K$ & $N$ \\
\hline 0,9 & 0,756 & 0,080 & 0,1270 & 13,32 & 0,673 & 0,255 & 1,031 & 1006 \\
\hline 0,8 & 0,700 & 0,100 & 0,0686 & 9,66 & 0,500 & 0,214 & 1,049 & 616 \\
\hline 0,7 & 0,630 & 0,110 & 0,0366 & 7,3 & 0,369 & 0,178 & 1,051 & 560 \\
\hline 0,6 & 0,550 & 0,100 & 0,0197 & 5,73 & 0,286 & 0,154 & 1,048 & 563 \\
\hline 0,5 & 0,460 & 0,100 & 0,0117 & 4,58 & 0,200 & 0,120 & 1,035 & 718 \\
\hline 0,4 & 0,374 & 0,089 & 0,0057 & 3,8 & 0,139 & 0,093 & 1,024 & 1004 \\
\hline 0,3 & 0,284 & 0,073 & 0,0025 & 3,18 & 0,091 & 0,067 & 1,014 & 1654 \\
\hline 0,2 & 0,191 & 0,053 & 0,0009 & 2,65 & 0,052 & 0,043 & 1,007 & 3587 \\
\hline 0,1 & 0,096 & 0,029 & 0,0002 & 2,17 & 0,022 & 0,020 & 1,002 & 15064 \\
\hline
\end{tabular}

\section{PROOFS OF LEMMAS}

Proof of Lemma 1. For $k=1, \ldots, n$, fix $\Re\left(z_{k}\right)$; then $\left|\delta-z_{k}\right|$ is maximum when $\left|z_{k}\right|=1$. Therefore, we can assume that $\left|z_{k}\right|=1$ and write $z_{k}=e^{i \theta_{k}}$. The mapping $\Phi(x)=\frac{1}{2} \log \left(1+\delta^{2}-2 \delta x\right)$ is concave. By Jensen's inequality we get

$$
\begin{aligned}
\log \left(\left|\prod_{k=1}^{n}\left(\delta-z_{k}\right)\right|^{1 / n}\right) & =\frac{1}{n} \sum_{k=1}^{n} \log \left|\delta-z_{k}\right| \\
& =\frac{1}{n} \sum_{k=1}^{n} \Phi\left(\cos \theta_{k}\right) \\
& \leq \Phi\left(\frac{1}{n} \sum_{k=1}^{n} \cos \theta_{k}\right) \\
& =\log \left(\sqrt{1+\delta^{2}-2 \delta s}\right)
\end{aligned}
$$

which establishes the lemma.

Proof of Lemma 2, For $k=1, \ldots, n-1$, if $\Re\left(\zeta_{k}\right)$ is given, the modulus $\left|\frac{\delta-\zeta_{k}}{a-\zeta_{k}}\right|$ is maximal when $\left|\zeta_{k}\right|=1$. Therefore, we can assume that for all $k,\left|\zeta_{k}\right|=1$. The mapping $\Phi$ defined on $[-1, a / 2]$ by

$$
\Phi(x)=\frac{1}{2} \log \left(\frac{1+\delta^{2}-2 \delta x}{1+a^{2}-2 a x}\right)
$$

is convex since

$$
\Phi^{\prime \prime}(x)=\frac{2(a-\delta)(1-a \delta)((a+\delta)(1+a \delta)-2 a \delta x)}{\left(1+\delta^{2}-2 \delta x\right)^{2}\left(1+a^{2}-2 a x\right)^{2}} \geq 0 .
$$


We deduce that

$$
\begin{aligned}
\log \left(\prod_{k=1}^{n-1}\left|\frac{\delta-\zeta_{k}}{a-\zeta_{k}}\right|\right) & =\sum_{k=1}^{n-1} \Phi\left(\operatorname{Re}\left(\zeta_{k}\right)\right) \\
& \leq(n-1)\left[q \Phi(-1)+(1-q) \Phi\left(\frac{a}{2}\right)\right] \\
& \leq(n-1)\left[q \log \left(\frac{1+\delta}{1+a}\right)+(1-q) \log \sqrt{1+\delta^{2}-\delta a}\right]
\end{aligned}
$$

where $q$ satisfies $-q+(1-q) \frac{a}{2}=s$, and so $q=\frac{a / 2-s}{1+a / 2}$. Applying the exponential function to both sides we obtain the lemma.

Proof of Lemma 3. Let $k \in\{1, \ldots, n-1\}$; for $\Re\left(\zeta_{k}\right)$ given, the modulus $\left|b-\zeta_{k}\right|$ is minimal when $\left|a-\zeta_{k}\right|=1$ or $\zeta_{k}$ is real with $-1 \leq \zeta_{k} \leq-1+a$. Assume that $\left|a-\zeta_{k}\right|=1$ or $\zeta_{k} \in[-1, a-1]$ for each $k$. The mapping $\Phi$, defined on $[-1, a / 2]$ by

$$
x \longmapsto\left\{\begin{array}{clc}
\frac{1}{2} \log (1+(b-a)(a+b-2 x)) & \text { if } & x \geq a-1, \\
\log (b-x) & \text { if } & x \in[-1, a-1],
\end{array}\right.
$$

satisfies $\Phi^{\prime \prime}(x) \leq 0$. Then $\Phi$ is concave on $[-1,-1+a]$ and on $[-1+a, a / 2]$. We deduce that

$$
\begin{aligned}
\log \left(\prod_{k=1}^{n-1}\left|b-\zeta_{k}\right|\right) & =\sum_{k=1}^{n-1} \Phi\left(\Re\left(\zeta_{k}\right)\right) \\
& \geq(n-1) \min \{\alpha \Phi(-1)+\beta \Phi(-1+a)+\gamma \Phi(a / 2)\},
\end{aligned}
$$

where the minimum is taken over the set of all $(\alpha, \beta, \gamma) \in \mathbb{R}_{+}^{3}$ satisfying

$$
\left\{\begin{array}{ccc}
\alpha+\beta+\gamma & = & 1 \\
-\alpha+(a-1) \beta+\frac{a}{2} \gamma & = & s
\end{array}\right.
$$

Let us define the mappings $g, f_{1}, f_{2}: \mathbb{R}_{+}^{3} \longrightarrow \mathbb{R}$ by

$$
\begin{aligned}
g(\alpha, \beta, \gamma) & =\alpha \log (b+1)+\beta \log (b+1-a)+\frac{\gamma}{2} \log \left(1+b^{2}-a b\right), \\
f_{1}(\alpha, \beta, \gamma) & =\alpha+\beta+\gamma \\
f_{2}(\alpha, \beta, \gamma) & =-\alpha+(-1+a) \beta+\frac{a}{2} \gamma
\end{aligned}
$$

Then, $\frac{1}{n-1} \log \left(\prod_{k=1}^{n-1}\left|b-\zeta_{k}\right|\right)$ is greater than

$$
\min _{(\alpha, \beta, \gamma) \in \mathbb{R}_{+}^{3}}\left\{g(\alpha, \beta, \gamma) ; f_{1}(\alpha, \beta, \gamma)=1 \text { and } f_{2}(\alpha, \beta, \gamma)=m\right\} \text {. }
$$

The Lagrange multipliers theory asserts that if the minimum is reached at $\left(\alpha_{0}, \beta_{0}, \gamma_{0}\right)$ $\in\left(\mathbb{R}_{+}^{*}\right)^{3}$, then there exist multipliers $\lambda_{1}$ and $\lambda_{2}$ such that

$$
\nabla g=\lambda_{1} \nabla f_{1}+\lambda_{2} \nabla f_{2}
$$

i.e.

$$
\left(\begin{array}{c}
\log (b+1) \\
\log (b+1-a) \\
\frac{1}{2} \log \left(1+b^{2}-a b\right)
\end{array}\right)=\lambda_{1}\left(\begin{array}{c}
1 \\
1 \\
1
\end{array}\right)+\lambda_{2}\left(\begin{array}{c}
-1 \\
-1+a \\
a / 2
\end{array}\right) \text {. }
$$

This is impossible in the generic case. We deduce (if necessary slightly modifying $a$ or $b)$ that if the minimum of (5) is reached at $(\alpha, \beta, \gamma)$, then we have $\alpha=0$ or $\beta=0$ or $\gamma=0$. Let us consider the different possibilities. 
- If $\alpha=0$, replacing in (4) we obtain $\beta=\frac{a / 2-s}{1-a / 2}$; thus

$$
\prod_{k=1}^{n-1}\left|b-b_{k}\right| \geq\left((b+1-a)^{\beta}\left(\sqrt{1+b^{2}-a b}\right)^{1-\beta}\right)^{n-1}=B_{1}^{n-1} \text {. }
$$

- If $\beta=0$, replacing in (44) we obtain $\alpha=\frac{a / 2-s}{1+a / 2}$; thus

$$
\prod_{k=1}^{n-1}\left|b-b_{k}\right| \geq\left((b+1)^{\alpha}\left({\sqrt{1+b^{2}-a b}}^{1-\alpha}\right)^{n-1}=B_{2}^{n-1} .\right.
$$

- If $\gamma=0$, we have

$$
\prod_{k=1}^{n-1}\left|b-b_{k}\right| \geq(1+b-a)^{n-1} \geq B_{1}^{n-1}
$$

This completes the proof of Lemma 3 .

Proof of Lemma 4. Let $k \in\{1, \ldots, n\}$. If $\left|\frac{c-z_{k}}{1-c z_{k}}\right|$ is given, then $\left|z_{k}\right|$ is maximal when $z_{k}$ is a real number with $\frac{c+r}{1+c r} \leq z_{k} \leq 1$. For $k=1, \ldots, n$, assume that $z_{k} \in$ $\left[\frac{c+r}{1+c r}, 1\right]$, write $\alpha_{k}=\log \left(z_{k}\right)$ and consider the mapping $\Phi$, defined on $\left[\log \left(\frac{c+r}{1+c r}\right), 0\right]$, by

$$
\Phi(\alpha)=\log \left(\frac{e^{\alpha}-c}{1-c e^{\alpha}}\right)
$$

It's easily seen that $\Phi$ is concave; therefore,

$$
\begin{aligned}
\log \left(\prod_{k=1}^{n}\left|\frac{c-z_{k}}{1-c z_{k}}\right|\right) & =\sum_{k=1}^{n} \Phi\left(\alpha_{k}\right) \\
& \geq \beta \Phi\left(\log \left(\frac{c+r}{1+c r}\right)\right)+(n-\beta) \Phi(0),
\end{aligned}
$$

where $\beta \log \left(\frac{c+r}{1+c r}\right)=\log \left(\prod_{k=1}^{n}\left|z_{k}\right|\right)$. Lemma 4 is obtained taking the exponential.

Proof of Lemma 5. The following inequalities are equivalent:

$$
\begin{aligned}
& |c-z| \geq \frac{c}{1-h}\left|\frac{(1-h)^{2}}{c}-z\right| \\
\Longleftrightarrow & \left|\frac{z}{1-h}-\frac{c}{1-h}\right|^{2} \geq\left|1-\frac{c}{1-h} \frac{z}{1-h}\right|^{2} \\
\Longleftrightarrow & \left|\frac{z}{1-h}\right|^{2}+\left(\frac{c}{1-h}\right)^{2} \geq 1+\left|\frac{c z}{(1-h)^{2}}\right|^{2} \\
\Longleftrightarrow & \left(\left|\frac{z}{1-h}\right|^{2}-1\right)\left(1-\left(\frac{c}{1-h}\right)^{2}\right) \geq 0 .
\end{aligned}
$$

We deduce the lemma. 
Proof of Lemma 6. Let us compute the quotient:

$$
\begin{aligned}
\left|\frac{P^{\prime}(b)}{P(b)}\right| & =\left|\frac{1}{b-a}+\sum_{k=1}^{n-1} \frac{1}{b-z_{k}}\right| \\
& \leq \frac{1}{b-a}+\sum_{k=1}^{n-1} \frac{1}{\left|b-z_{k}\right|} \\
& \leq \frac{n}{b-1} .
\end{aligned}
$$

Then $|P(b)| \geq \frac{b-1}{n}\left|P^{\prime}(b)\right|=(b-1) \prod_{k=1}^{n-1}\left|b-\zeta_{k}\right|$, and the lemma follows.

Proof of Lemma 7, Set $k=\frac{c(a-c)}{2\left((1-h)^{2}-c^{2}\right)}$; the disk $\mathcal{D}$ has center $\omega$ and radius $R$ given by

$$
\omega=c \frac{1-k^{2}(1-h)^{2}}{1-(k c)^{2}} \quad \text { and } \quad R=k \frac{(1-h)^{2}-c^{2}}{1-(k c)^{2}} .
$$

According to Theorem 3, it suffices to show that

$$
R \leq 1-\sqrt{1+\omega^{2}-\omega a}
$$

or equivalently that

$$
\omega^{2}-R^{2} \leq \omega a-2 R \quad \text { and } \quad R \leq 1 .
$$

The first inequality holds since

$$
\omega^{2}-R^{2} \leq \omega a-2 R \quad \Longleftrightarrow \quad k^{2}(1-h)^{2}\left((1-h)^{2}-a c\right) \geq 0 .
$$

On the other hand,

$$
R \leq 1 \quad \Longleftrightarrow \quad \frac{c(a-c)}{2} \leq 1-k^{2} c^{2} .
$$

Therefore the second inequality is straightforward since $k \leq \frac{1}{2}$. The lemma is proved.

\section{Conclusion}

It may be surprising to see that Sendov's conjecture is easily proved in extremal cases, meaning when $a=0$ or $a=1$ and in the generic case $0<a<1$, only a few partial results are known. In the present paper, we try to fill this gap, but it remains to obtain a definitive proof of the conjecture, that is, to demonstrate that, with our notations, $N=8$ for all $a \in(0,1)$.

We have shown that if a zero, denoted by $a$, of $P$ is given, one can compute an integer bound $N$ such that if $\operatorname{deg} P \geq N$, then $P^{\prime}$ has a zero in the disk $|z-a| \leq 1$. It would be nice if $N$ could be given independently of $|a|$ or, at least, to have an explicit formula for $N$ in terms of $a$.

\section{ACKNOWLEDGEMENT}

The author would like to thank an anonymous referee for a careful reading of the manuscript, valuable suggestions and detailed advice. 


\section{REFERENCES}

[1] Johnny E. Brown and Guangping Xiang, Proof of the Sendov conjecture for polynomials of degree at most eight, J. Math. Anal. Appl. 232 (1999), no. 2, 272-292, DOI 10.1006/jmaa.1999.6267. MR.1683144 (2001b:30007)

[2] J. H. Grace, The zeros of a polynomial, Proc. Cambridge Philos. Soc. 11, 1902, 352-357.

[3] W. K. Hayman, Research problems in function theory, The Athlone Press, University of London, London, 1967. MR.0217268 (36 \#359)

[4] Morris Marden, Conjectures on the critical points of a polynomial, Amer. Math. Monthly 90 (1983), no. 4, 267-276, DOI 10.2307/2975758. MR700266 (84e:30007)

[5] Q. I. Rahman and G. Schmeisser, Analytic theory of polynomials, London Mathematical Society Monographs. New Series, vol. 26, The Clarendon Press, Oxford University Press, Oxford, 2002. MR:1954841 (2004b:30015)

[6] Zalman Rubinstein, On a problem of Ilyeff, Pacific J. Math. 26 (1968), 159-161. MR.0237753 (38 \#6034)

[7] Gerhard Schmeisser, Bemerkungen zu einer Vermutung von Ilieff (German), Math. Z. 111 (1969), 121-125. MR0264040(41 \#8637)

[8] Blagovest Sendov, On the critical points of a polynomial, East J. Approx. 1 (1995), no. 2, 255-258. MR 1391770 (97k:30007)

LycÉe Louis-Le-Grand, 123 Rue St JaCques, 75005 PARis, France

E-mail address: jerome.degot@numericable.fr 